\title{
New constructions of MDS codes with complementary duals
}

\author{
Bocong Chen ${ }^{1}$, Hongwei $\mathrm{Liu}^{2}$ \\ ${ }^{1}$ School of Mathematics, South China University of Technology, Guangzhou, Guangdong, 510641, China \\ ${ }^{2}$ School of Mathematics and Statistics, Central China Normal University, Wuhan, Hubei, 430079, China
}

\begin{abstract}
Linear complementary-dual (LCD for short) codes are linear codes that intersect with their duals trivially. LCD codes have been used in certain communication systems. It is recently found that LCD codes can be applied in cryptography. This application of LCD codes renewed the interest in the construction of LCD codes having a large minimum distance. MDS codes are optimal in the sense that the minimum distance cannot be improved for given length and code size. Constructing LCD MDS codes is thus of significance in theory and practice. Recently, Jin ( $[$ ], IEEE Trans. Inf. Theory, 2016) constructed several classes of LCD MDS codes through generalized Reed-Solomon codes. In this paper, a different approach is proposed to obtain new LCD MDS codes from generalized ReedSolomon codes. Consequently, new code constructions are provided and certain previously known results in 8 ] are extended.
\end{abstract}

Keywords: Linear complementary dual, generalized Reed-Solomon code, MDS code.

2010 Mathematics Subject Classification: 94B05, $11 \mathrm{~T} 71$.

\section{Introduction}

A linear complementary-dual (LCD for short) code is a linear code $\mathcal{C}$ that intersects with its dual $\mathcal{C}^{\perp}$ trivially, i.e., $\mathcal{C} \cap \mathcal{C}^{\perp}=\{\mathbf{0}\}$. This class of codes was introduced by Massey [15, where he showed that asymptotically good LCD codes exist. In a follow-up paper [18, Yang and Massey gave a necessary and sufficient condition for a cyclic code to be an LCD code. Necessary and sufficient conditions for certain classes of quasi-cyclic codes to be LCD codes were obtained in [5. Dinh [3] proved that any $\lambda$-constayclic codes with $\lambda \notin\{1,-1\}$ are LCD codes. Güneri et al. 6] studied LCD quasi-cyclic codes, including the algebraic characterizations, the asymptotic properties and the code constructions. Dougherty et al. 4] presented a linear programming bound on the largest size of an LCD code of given length and minimum distance. The parameters of several classes of LCD BCH codes were explicitly determined in [10, 11, 12. Hou and Oggier [7] established a corresponding relationship between lattices and LCD codes.

LCD codes have been used in certain communication systems. Recently, Carlet and Guilley [2] found that LCD codes can be applied in cryptography. This application of LCD codes renewed the interest in the construction of LCD codes having a large minimum distance. Maximum distance separable (MDS) codes are optimal in the sense that no code of length $n$ with $M$ codewords has a larger minimum distance than an MDS code with length $n$ and size $M$. Constructing LCD MDS codes is thus of significance in theory and practice. The class of generalized Reed-Solomon (GRS) codes is probably the best known family of MDS codes. Therefore, it is natural to construct LCD MDS codes through GRS codes. Recently, Jin 8] constructed several classes of LCD MDS codes by using two classes of disjoint GRS codes. The existence question about LCD MDS codes over a finite field of even characteristic has been completely addressed in [8, Theorem IV.2]. Some other constructions of LCD MDS codes are known, e.g., see [16], [17] and [19].

The purpose of this paper is to find new LCD MDS codes from GRS codes or extended GRS codes. By virtue of [8, Theorem IV.2], we only consider the construction of LCD MDS codes over a finite field with

Email addresses: bocong_chen@yahoo.com (B. Chen), hwliu@mail.ccnu.edu.cn (H. Liu). 
odd characteristic. A different approach from [8] is proposed to obtain new LCD MDS codes; specifically, some results of 8 are extended. Note that the dual of an LCD code is an LCD code again. As a consequence, we always restrict ourself to $k$-dimensional codes of length $n$ with $1<k \leq\lfloor n / 2\rfloor$, where $\lfloor a\rfloor$ denotes the integer part of a real number $a$. The main results of this paper are summarized as follows.

Theorem 1.1. Let $q>3$ be an odd prime power. Let $n>1$ and $k$ be positive integers with $1<k \leq\lfloor n / 2\rfloor$. Then there exists a q-ary $[n, k] L C D M D S$ code whenever one of the following conditions holds.

(1) (see Theorem 3.3) $n=q+1$.

(2) (see Theorem 3.4) $n>1$ is a divisor of $q-1$.

(3) (see Theorem 3.5) $q=p^{e}$ and $n=p^{\ell}$, where $p$ is prime and $1 \leq \ell \leq e$.

(4) (see Theorem [3.6) $n<q$ and $n+k \geq q+1$.

(5) (see Theorem 3.7) $n<q$ and $2 n-k<q \leq 2 n$.

At this point we make several remarks. The first part of Theorem 1.1 improves [8, Theorem IV.4] by removing the even $k$ constraint, and the existence question about LCD MDS codes of length $n=q+1$ is completely addressed. The second and the third parts, respectively, say that $k$-dimensional LCD MDS codes of length $q-1$ and $q$ over $\mathbb{F}_{q}$ exist. The last two parts of Theorem 1.1 imply that $[n, k]$ LCD MDS codes exist if $n$ and $k$ are close to $q$. The conclusions of our results are different from those in [8, Theorem IV.3] and [8, Theorem IV.6], where it requires that the value of $q$ is much bigger than the value of $n$.

The remainder of this paper is organized as follows. Basic notations and results about GRS codes and extended GRS codes are provided in Section 2, The main results included in Theorem 1.1 are presented in Section 3 ,

\section{Preliminaries}

In this section, we review some basic notations and results about generalized Reed-Solomon codes and extended generalized Reed-Solomon codes. For the details, the reader is referred to [13] or [14]. Let $\mathbb{F}_{q}$ be the finite field of order $q$, let $n$ be a positive integer with $1<n \leq q$, and let $\mathbf{a}=\left(\alpha_{1}, \alpha_{2}, \cdots, \alpha_{n}\right)$, where $\alpha_{i}(1 \leq i \leq n)$ are distinct elements of $\mathbb{F}_{q}$. Fix $n$ nonzero elements $v_{1}, v_{2}, \cdots, v_{n}$ of $\mathbb{F}_{q}\left(v_{i}\right.$ are not necessarily distinct). For $1 \leq k \leq n$, the $k$-dimensional generalized Reed-Solomon code (GRS code for short) of length $n$ associated with $\mathbf{a}=\left(\alpha_{1}, \alpha_{2}, \cdots, \alpha_{n}\right)$ and $\mathbf{v}=\left(v_{1}, v_{2}, \cdots, v_{n}\right)$ is defined to be

$$
G R S_{k}(\mathbf{a}, \mathbf{v})=\left\{\left(v_{1} f\left(\alpha_{1}\right), v_{2} f\left(\alpha_{2}\right), \cdots, v_{n} f\left(\alpha_{n}\right)\right) \mid f(X) \in \mathbb{F}_{q}[X], \operatorname{deg} f(X) \leq k-1\right\} .
$$

Clearly, $G R S_{k}(\mathbf{a}, \mathbf{v})$ has a generator matrix

$$
G=\left(\begin{array}{cccc}
v_{1} & v_{2} & \cdots & v_{n} \\
v_{1} \alpha_{1} & v_{2} \alpha_{2} & \cdots & v_{n} \alpha_{n} \\
v_{1} \alpha_{1}^{2} & v_{2} \alpha_{2}^{2} & \cdots & v_{n} \alpha_{n}^{2} \\
\vdots & \vdots & \ddots & \vdots \\
v_{1} \alpha_{1}^{k-1} & v_{2} \alpha_{2}^{k-1} & \cdots & v_{n} \alpha_{n}^{k-1}
\end{array}\right) .
$$

It is well known that the code $G R S_{k}(\mathbf{a}, \mathbf{v})$ is a $q$-ary $[n, k, n-k+1]$-MDS code and the dual of a GRS code is again a GRS code; indeed,

$$
G R S_{k}(\mathbf{a}, \mathbf{v})^{\perp}=G R S_{n-k}\left(\mathbf{a}, \mathbf{v}^{\prime}\right)
$$

for some $\mathbf{v}^{\prime}=\left(v_{1}^{\prime}, v_{2}^{\prime}, \cdots, v_{n}^{\prime}\right)$ with $v_{i}^{\prime} \neq 0$ for all $1 \leq i \leq n$ (e.g., see [13] or [14). The vector $\mathbf{v}^{\prime}$ can be chosen as any vector that generates the dual of $G R S_{n-1}(\mathbf{a}, \mathbf{v})$. 
Let 1 denote the all-one row vector with appropriate length. The dual of $G R S_{k}(\mathbf{a}, \mathbf{1})$ is $G R S_{n-k}(\mathbf{a}, \mathbf{u})$, where $\mathbf{u}=\left(u_{1}, u_{2}, \cdots, u_{n}\right)$ with $u_{i}=\prod_{1 \leq j \leq n, j \neq i}\left(\alpha_{i}-\alpha_{j}\right)^{-1}$ for $1 \leq i \leq n$ (e.g., see [9, Lemma 2.3]). More precisely, one has

$$
G R S_{k}(\mathbf{a}, \mathbf{1})^{\perp}=G R S_{n-k}(\mathbf{a}, \mathbf{u})=\left\{\left(u_{1} g\left(\alpha_{1}\right), \cdots, u_{n} g\left(\alpha_{n}\right)\right) \mid g(X) \in \mathbb{F}_{q}[X], \operatorname{deg} g(X) \leq n-k-1\right\} .
$$

GRS codes are probably the best known family of MDS codes. Obviously, GRS codes exist for any length $n \leq q$ and any dimension $k \leq n$. GRS codes of length $n$ can be extended to codes of length $n+1$ while preserving the MDS property by appending to $G$ an extra column of the form $(0,0, \cdots, 0, \beta)^{T}$ with $\beta$ being a nonzero element of $\mathbb{F}_{q}$. In this paper, we consider extended GRS codes of length $q+1$. Label the elements of $\mathbb{F}_{q}=\left\{\alpha_{1}, \alpha_{2}, \cdots, \alpha_{q}\right\}$. Given a vector $\mathbf{v}=\left(v_{1}, v_{2}, \cdots, v_{q}\right)$ with $v_{j} \neq 0$ for all $1 \leq j \leq q$, the $k$-dimensional extended $G R S$ code of length $q+1$ associated with $\mathbf{a}=\left(a_{1}, a_{2}, \cdots, a_{q}\right)$ and $\mathbf{v}$ is defined as

$$
G R S_{k}(\mathbf{a}, \mathbf{v}, \infty)=\left\{\left(v_{1} f\left(\alpha_{1}\right), \cdots, v_{q} f\left(\alpha_{q}\right), f_{k-1}\right) \mid f(X) \in \mathbb{F}_{q}[X], \operatorname{deg} f(X) \leq k-1\right\},
$$

where $f_{k-1}$ is the coefficient of $X^{k-1}$ of the polynomial $f(X)$. It is true that $G R S_{k}(\mathbf{a}, \mathbf{v}, \infty)$ is a $q$-ary MDS code with parameters $[q+1, k, q-k+2]$ (e.g., see [13] or [14]). The code $G R S_{k}(\mathbf{a}, \mathbf{v}, \infty)$ has a generator matrix

$$
G_{\infty}=\left(\begin{array}{ccccc}
v_{1} & v_{2} & \cdots & v_{q} & 0 \\
v_{1} \alpha_{1} & v_{2} \alpha_{2} & \cdots & v_{q} \alpha_{q} & 0 \\
v_{1} \alpha_{1}^{2} & v_{2} \alpha_{2}^{2} & \cdots & v_{q} \alpha_{q}^{2} & 0 \\
\vdots & \vdots & \ddots & \vdots & \vdots \\
v_{1} \alpha_{1}^{k-1} & v_{2} \alpha_{2}^{k-1} & \cdots & v_{q} \alpha_{q}^{k-1} & 1
\end{array}\right) .
$$

The dual of $G R S_{k}(\mathbf{a}, \mathbf{1}, \infty)$ can be determined explicitly; in fact, it is not hard to verify that the dual of $G R S_{k}(\mathbf{a}, \mathbf{1}, \infty)$ is

$$
G R S_{k}(\mathbf{a}, \mathbf{1}, \infty)^{\perp}=\left\{\left(g\left(\alpha_{1}\right), g\left(\alpha_{2}\right), \cdots, g\left(\alpha_{q}\right), g_{q-k}\right) \mid g(X) \in \mathbb{F}_{q}[X], \operatorname{deg} g(X) \leq q-k\right\},
$$

where $g_{q-k}$ is the coefficient of $X^{q-k}$ of the polynomial $g(X)$ (e.g., see [1, Lemma 7.7] or [9, Lemma 2.3]).

\section{Constructions of LCD MDS codes}

The purpose of this section is to find LCD codes among the family of GRS codes or among the family of extended GRS codes. Consequently the resulting codes are simultaneously LCD and MDS. As mentioned in the first section, the existence question about LCD MDS codes over a finite field of even characteristic has been completely addressed in [8]. We therefore only consider GRS codes over a finite field with odd characteristic.

Note that the dual of an LCD code is an LCD code again. As a consequence, we always restrict ourself to $k$-dimensional codes of length $n$ with $1<k \leq\lfloor n / 2\rfloor$, where $\lfloor a\rfloor$ denotes the integer part of a real number $a$.

We begin with the following lemma which is useful for the construction of LCD GRS codes.

Lemma 3.1. Suppose $G R S_{k}(\mathbf{a}, \mathbf{v})$ is the GRS code associated with a and $\mathbf{v}$, as in (2.1). A typical codeword $\mathbf{c}=\left(v_{1} f\left(\alpha_{1}\right), v_{2} f\left(\alpha_{2}\right), \cdots, v_{n} f\left(\alpha_{n}\right)\right)$ of $G R S_{k}(\mathbf{a}, \mathbf{v})$ is contained in $G R S_{k}(\mathbf{a}, \mathbf{v})^{\perp}$ if and only if a polynomial $g(X) \in \mathbb{F}_{q}[X]$ with $\operatorname{deg} g(X) \leq n-k-1$ can be found such that

$$
\left(v_{1}^{2} f\left(\alpha_{1}\right), v_{2}^{2} f\left(\alpha_{2}\right), \cdots, v_{n}^{2} f\left(\alpha_{n}\right)\right)=\left(u_{1} g\left(\alpha_{1}\right), u_{2} g\left(\alpha_{2}\right), \cdots, u_{n} g\left(\alpha_{n}\right)\right),
$$

where $u_{i}=\prod_{1 \leq j \leq n, j \neq i}\left(\alpha_{i}-\alpha_{j}\right)^{-1}$ for $1 \leq i \leq n$. 
Proof. Note that $G R S_{k}(\mathbf{a}, \mathbf{v})$ has a generator matrix $G$ given by (2.2). Clearly, we have $G=G_{1} \Delta$, where

$$
G_{1}=\left(\begin{array}{cccc}
1 & 1 & \cdots & 1 \\
\alpha_{1} & \alpha_{2} & \cdots & \alpha_{n} \\
\alpha_{1}^{2} & \alpha_{2}^{2} & \cdots & \alpha_{n}^{2} \\
\vdots & \vdots & \ddots & \vdots \\
\alpha_{1}^{k-1} & \alpha_{2}^{k-1} & \cdots & \alpha_{n}^{k-1}
\end{array}\right)
$$

and $\Delta$ is the diagonal matrix

$$
\Delta=\left(\begin{array}{llll}
v_{1} & & & \\
& v_{2} & & \\
& & \ddots & \\
& & & v_{n}
\end{array}\right) .
$$

It follows that $\mathbf{c}=\left(v_{1} f\left(\alpha_{1}\right), v_{2} f\left(\alpha_{2}\right), \cdots, v_{n} f\left(\alpha_{n}\right)\right)$ is contained in $G R S_{k}(\mathbf{a}, \mathbf{v})^{\perp}$ if and only if

$$
G \mathbf{c}^{T}=\left(G_{1} \Delta\right) \mathbf{c}^{T}=G_{1}\left(\Delta \mathbf{c}^{T}\right)=G_{1}\left(v_{1}^{2} f\left(\alpha_{1}\right), v_{2}^{2} f\left(\alpha_{2}\right), \cdots, v_{n}^{2} f\left(\alpha_{n}\right)\right)^{T}=\mathbf{0},
$$

where $\mathbf{c}^{T}$ denotes the transpose of $\mathbf{c}$. Recall that the dual of $G R S_{k}(\mathbf{a}, \mathbf{1})$ is $G R S_{n-k}(\mathbf{a}, \mathbf{u})$, where $\mathbf{u}=\left(u_{1}, u_{2}, \cdots, u_{n}\right)$ with $u_{i}=\prod_{1 \leq j \leq n, j \neq i}\left(\alpha_{i}-\alpha_{j}\right)^{-1}$ for $1 \leq i \leq n$. Now the desired result follows immediately from (2.3).

The arguments used in Lemma 3.1 can be modified to obtain an analogous result for extended GRS codes.

Lemma 3.2. Suppose $G R S_{k}(\mathbf{a}, \mathbf{v}, \infty)$ is the extended $G R S$ code associated with $\mathbf{a}=\left(\alpha_{1}, \alpha_{2}, \cdots, \alpha_{q}\right)$ and $\mathbf{v}=\left(v_{1}, v_{2}, \cdots, v_{q}\right)$, as in 2.4). A typical codeword $\mathbf{c}=\left(v_{1} f\left(\alpha_{1}\right), \cdots, v_{q} f\left(\alpha_{q}\right), f_{k-1}\right)$ of $G R S_{k}(\mathbf{a}, \mathbf{v}, \infty)$ is contained in $G R S_{k}(\mathbf{a}, \mathbf{v}, \infty)^{\perp}$ if and only if a polynomial $g(X) \in \mathbb{F}_{q}[X]$ with $\operatorname{deg} g(X) \leq q-k$ can be found such that

$$
\left(v_{1}^{2} f\left(\alpha_{1}\right), \cdots, v_{q}^{2} f\left(\alpha_{q}\right), f_{k-1}\right)=\left(g\left(\alpha_{1}\right), \cdots, g\left(\alpha_{q}\right), g_{q-k}\right),
$$

where $g_{q-k}$ is the coefficient of $X^{q-k}$ of the polynomial $g(X)$.

We first construct LCD MDS codes of length $q+1$ by using Lemma 3.2, 8, Theorem IV.4] guarantees that there exists a $k$-dimensional LCD MDS code of length $q+1$ when $k$ is even. The next result improves [8, Theorem IV.4] by removing the even $k$ constraint. Recall that we always restrict ourself to $k$-dimensional codes of length $n$ with $1<k \leq\lfloor n / 2\rfloor$.

Theorem 3.3. Let $q>3$ be an odd prime power. Then there exists a $k$-dimensional LCD extended GRS code of length $q+1$ over $\mathbb{F}_{q}$.

Proof. Label the elements of $\mathbb{F}_{q}=\left\{\alpha_{1}, \alpha_{2}, \cdots, \alpha_{q}\right\}$. Let $\mathbf{a}=\left(\alpha_{1}, \alpha_{2}, \cdots, \alpha_{q}\right)$ and let $\gamma$ be a primitive $(q-1)$ th root of unity in $\mathbb{F}_{q}$, i.e., $q-1$ is the smallest positive integer such that $\gamma^{q-1}=1$. Since $q>3$, we have $\gamma^{2} \neq 1$.

We now consider two cases separately.

Case 1: $2 \leq k<(q+1) / 2$. Let

$$
\mathbf{v}=\left(v_{1}, v_{2}, \cdots, v_{q-k+1}, v_{q-k+2}, \cdots, v_{q}\right),
$$

where $v_{i}=1$ for $1 \leq i \leq q-k+1$ and $v_{i}=\gamma$ for $q-k+2 \leq i \leq q$. Consider the extended GRS code $G R S_{k}(\mathbf{a}, \mathbf{v}, \infty)$ of length $q+1$ over $\mathbb{F}_{q}$ associated with $\mathbf{a}$ and $\mathbf{v}$, i.e.,

$$
\begin{aligned}
G R S_{k}(\mathbf{a}, \mathbf{v}, \infty)=\{ & \left(f\left(\alpha_{1}\right), f\left(\alpha_{2}\right), \cdots, f\left(\alpha_{q-k+1}\right),\right. \\
& \left.\left.\gamma f\left(\alpha_{q-k+2}\right), \cdots, \gamma f\left(\alpha_{q}\right), f_{k-1}\right) \mid f(X) \in \mathbb{F}_{q}[X], \operatorname{deg} f(X) \leq k-1\right\},
\end{aligned}
$$


where $f_{k-1}$ is the coefficient of $X^{k-1}$ of the polynomial $f(X)$. We now aim to show that $G R S_{k}(\mathbf{a}, \mathbf{v}, \infty)$ is an LCD code, namely $G R S_{k}(\mathbf{a}, \mathbf{v}, \infty) \bigcap G R S_{k}(\mathbf{a}, \mathbf{v}, \infty)^{\perp}=\{\mathbf{0}\}$. For this purpose, let

$$
\mathbf{c}=\left(f\left(\alpha_{1}\right), f\left(\alpha_{2}\right), \cdots, f\left(\alpha_{q-k+1}\right), \gamma f\left(\alpha_{q-k+2}\right), \cdots, \gamma f\left(\alpha_{q}\right), f_{k-1}\right)
$$

be an arbitrary element of $G R S_{k}(\mathbf{a}, \mathbf{v}, \infty) \bigcap G R S_{k}(\mathbf{a}, \mathbf{v}, \infty)^{\perp}$, where $f(X) \in \mathbb{F}_{q}[X]$ with $\operatorname{deg} f(X) \leq k-1$. Applying Lemma 3.2, we see that a polynomial $g(X) \in \mathbb{F}_{q}[X]$ with $\operatorname{deg} g(X) \leq q-k$ can be found such that

$$
\begin{aligned}
& \left(f\left(\alpha_{1}\right), \cdots, f\left(\alpha_{q-k+1}\right), \gamma^{2} f\left(\alpha_{q-k+2}\right), \cdots, \gamma^{2} f\left(\alpha_{q}\right), f_{k-1}\right) \\
& =\left(g\left(\alpha_{1}\right), \cdots, g\left(\alpha_{q-k+1}\right), g\left(\alpha_{q-k+2}\right), \cdots, g\left(\alpha_{q}\right), g_{q-k}\right),
\end{aligned}
$$

where $g_{q-k}$ is the coefficient of $X^{q-k}$ of the polynomial $g(X)$. Our task is to show $\mathbf{c}=\mathbf{0}$, or equivalently $f(X)=0$. By $\operatorname{deg} f(X) \leq k-1 \leq q-k$ and $\operatorname{deg} g(X) \leq q-k$, (3.2) suggests $f(X)=g(X)$, since $f\left(\alpha_{i}\right)=g\left(\alpha_{i}\right)$ for $1 \leq i \leq q-k+1$. However, the last coordinate of Equation (3.2) requires $f_{k-1}=g_{q-k}$. This leads to $f_{k-1}=0$; otherwise we would have $k=(q+1) / 2$, a contradiction. Thus, $\operatorname{deg} f(X) \leq k-2$. At the moment, Equation (3.2) also gives

$$
\gamma^{2} f\left(\alpha_{j}\right)=g\left(\alpha_{j}\right)=f\left(\alpha_{j}\right) \text { for } q-k+2 \leq j \leq q,
$$

where the last equality holds because $f(X)=g(X)$. It follows from $\gamma^{2} \neq 1$ that $f\left(\alpha_{j}\right)=0$ for $q-k+2 \leq$ $j \leq q$. This forces $f(X)=0$, because $f(X) \in \mathbb{F}_{q}[X]$ is a polynomial with $\operatorname{deg} f(X) \leq k-2$ and has $k-1$ distinct roots $\alpha_{q-k+2}, \cdots, \alpha_{q}$. We thus conclude $\mathbf{c}=\mathbf{0}$, as desired.

Case 2: $k=(q+1) / 2$. In this case, let

$$
\mathbf{v}^{\prime}=\left(v_{1}, v_{2}, \cdots, v_{k-1}, v_{k}, \cdots, v_{q}\right),
$$

where $v_{i}=1$ for $1 \leq i \leq k-1$ and $v_{i}=\gamma$ for $k \leq i \leq q$. Consider the extended GRS code $G R S_{k}\left(\mathbf{a}, \mathbf{v}^{\prime}, \infty\right)$ of length $q+1$ over $\mathbb{F}_{q}$ associated with $\mathbf{a}$ and $\mathbf{v}^{\prime}$, i.e.,

$$
\begin{aligned}
G R S_{k}\left(\mathbf{a}, \mathbf{v}^{\prime}, \infty\right)=\{ & \left(f\left(\alpha_{1}\right), f\left(\alpha_{2}\right), \cdots, f\left(\alpha_{k-1}\right),\right. \\
& \left.\left.\gamma f\left(\alpha_{k}\right), \cdots, \gamma f\left(\alpha_{q}\right), f_{k-1}\right) \mid f(X) \in \mathbb{F}_{q}[X], \operatorname{deg} f(X) \leq k-1\right\},
\end{aligned}
$$

where $f_{k-1}$ is the coefficient of $X^{k-1}$ of the polynomial $f(X)$. Let

$$
\left(f\left(\alpha_{1}\right), f\left(\alpha_{2}\right), \cdots, f\left(\alpha_{k-1}\right), \gamma f\left(\alpha_{k}\right), \cdots, \gamma f\left(\alpha_{q}\right), f_{k-1}\right)
$$

be an arbitrary element of $G R S_{k}\left(\mathbf{a}, \mathbf{v}^{\prime}, \infty\right)$ with $\operatorname{deg} f(X) \leq k-1=(q-1) / 2$. Therefore, we have a polynomial $g(X) \in \mathbb{F}_{q}[X]$ with $\operatorname{deg} g(X) \leq q-k=(q-1) / 2$ such that

$$
\left(f\left(\alpha_{1}\right), \cdots, f\left(\alpha_{k-1}\right), \gamma^{2} f\left(\alpha_{k}\right), \cdots, \gamma^{2} f\left(\alpha_{q}\right), f_{k-1}\right)=\left(g\left(\alpha_{1}\right), \cdots, g\left(\alpha_{k-1}\right), g\left(\alpha_{k}\right), \cdots, g\left(\alpha_{q}\right), g_{q-k}\right),
$$

where $g_{q-k}$ is the coefficient of $X^{q-k}$ of the polynomial $g(X)$. By Equation (3.3), one has $f_{k-1}=g_{q-k}$ which implies that $\operatorname{deg}(f(X)-g(X)) \leq k-2$. Similar arguments as in Case 1 give $f(X)=0$. We are done.

Next we turn to construct LCD MDS codes from GRS codes of length $1<n \leq q$. We obtain the following theorems.

Theorem 3.4. Let $q>3$ be an odd prime power. If $n>1$ is a divisor of $q-1$, then there exists a $k$-dimensional LCD GRS code of length $n$ over $\mathbb{F}_{q}$.

Proof. Since $n>1$ is a divisor of $q-1$, there exists a primitive $n$th root of unity $\omega$ in $\mathbb{F}_{q}$. Take $\mathbf{a}=\left(\omega^{0}, \omega^{1}, \cdots, \omega^{n-1}\right)$ and let $\mathbf{v}=\left(v_{1}, \cdots, v_{n-k+1}, v_{n-k+2}, \cdots, v_{n}\right)$, where $v_{i}=1$ for $1 \leq i \leq n-k+1$ and $v_{i} \notin\{-1,0,1\}$ for $n-k+2 \leq i \leq n$. Consider the GRS code $\mathcal{C}$ of length $n$ over $\mathbb{F}_{q}$ associated with $\mathbf{a}$ and $\mathbf{v}$ as follows

$$
\mathcal{C}=\left\{\left(f\left(\omega^{0}\right), \cdots, f\left(\omega^{n-k}\right), v_{n-k+2} f\left(\omega^{n-k+1}\right), \cdots, v_{n} f\left(\omega^{n-1}\right)\right) \mid f(X) \in \mathbb{F}_{q}[X], \operatorname{deg} f(X) \leq k-1\right\} .
$$


We claim that $\mathcal{C} \cap \mathcal{C}^{\perp}=\{\boldsymbol{0}\}$. To see this, let

$$
\left(f\left(\omega^{0}\right), \cdots, f\left(\omega^{n-k}\right), v_{n-k+2} f\left(\omega^{n-k+1}\right), \cdots, v_{n} f\left(\omega^{n-1}\right)\right)
$$

be an arbitrary element of $\mathcal{C} \cap \mathcal{C}^{\perp}$. Consequently,

$$
G_{2}\left(f\left(\omega^{0}\right), \cdots, f\left(\omega^{n-k}\right), v_{n-k+2}^{2} f\left(\omega^{n-k+1}\right), \cdots, v_{n}^{2} f\left(\omega^{n-1}\right)\right)^{T}=\mathbf{0},
$$

where $G_{2}$ is the $k \times n$ matrix

$$
G_{2}=\left(\begin{array}{cccc}
1 & 1 & \cdots & 1 \\
1 & \omega & \cdots & \omega^{n-1} \\
\vdots & \vdots & \ddots & \vdots \\
1 & \omega^{k-1} & \cdots & \omega^{(k-1)(n-1)}
\end{array}\right)
$$

At this point, it is easy to see that

$$
\left(\begin{array}{cccc}
1 & 1 & \cdots & 1 \\
1 & \omega & \cdots & \omega^{n-1} \\
\vdots & \vdots & \ddots & \vdots \\
1 & \omega^{n-2} & \cdots & \omega^{(n-2)(n-1)}
\end{array}\right)\left(\begin{array}{c}
1 \\
\omega \\
\vdots \\
\omega^{n-1}
\end{array}\right)=\mathbf{0} .
$$

This implies that a polynomial $g(X) \in \mathbb{F}_{q}[X]$ with $\operatorname{deg} g(X) \leq n-k-1$ can be found such that

$$
\left(f\left(\omega^{0}\right), \cdots, f\left(\omega^{n-k}\right), v_{n-k+2}^{2} f\left(\omega^{n-k+1}\right), \cdots, v_{n}^{2} f\left(\omega^{n-1}\right)\right)=\left(g\left(\omega^{0}\right), \omega g\left(\omega^{1}\right), \cdots, \omega^{n-1} g\left(\omega^{n-1}\right)\right) .
$$

The first $n-k+1$ coordinates of (3.4) give $f\left(\omega^{i}\right)=\omega^{i} g\left(\omega^{i}\right)$ for $0 \leq i \leq n-k$. Since $k \leq\lfloor n / 2\rfloor$, $\operatorname{deg} f(X) \leq k-1 \leq n-k-1$ and $\operatorname{deg} g(X) \leq n-k-1$, we have $f(X)=X g(X)$. In particular, $\operatorname{deg} g(X) \leq k-2$. By the last $k-1$ coordinates of (3.4), we have that for any $n-k+2 \leq j \leq n$,

$$
v_{j}^{2} f\left(\omega^{j-1}\right)=v_{j}^{2} \omega^{j-1} g\left(\omega^{j-1}\right)=\omega^{j-1} g\left(\omega^{j-1}\right) .
$$

It follows from $v_{j}^{2} \neq 1$ that $g\left(\omega^{j-1}\right)=0$. In other words, $g(X)$ has $k-1$ distinct roots, giving $g(X)=0$. Thus, $f(X)=0$. The proof is complete.

The following theorem particularly indicates that $k$-dimensional LCD MDS codes of length $q$ over $\mathbb{F}_{q}$ exist for any $1<k<q$.

Theorem 3.5. Let $q=p^{e}>3$, where $p$ is an odd prime number and $e \geq 1$ is an integer. Then there exists a $k$-dimensional $L C D$ GRS code of length $n=p^{\ell}$ over $\mathbb{F}_{q}$, where $\ell$ is a positive integer with $1 \leq \ell \leq e$.

Proof. Let $H$ be an additive subgroup of $\mathbb{F}_{p^{e}}$ of order $n=p^{\ell}$, say $H=\left\{\alpha_{1}, \cdots, \alpha_{n}\right\}$. Let $h$ be the product of all nonzero elements of $H$, namely

$$
h=\prod_{z \in H \backslash\{0\}} z .
$$

Take $\gamma \in \mathbb{F}_{q}^{*}$ with $\gamma^{2} \neq 1$. Let $\mathbf{a}=\left(\alpha_{1}, \alpha_{2}, \cdots, \alpha_{n}\right)$ and let

$$
\mathbf{v}=\left(v_{1}, \cdots, v_{n-k}, v_{n-k+1}, \cdots, v_{n}\right),
$$

where $v_{i}=1$ for $1 \leq i \leq n-k$ and $v_{i}=\gamma$ for $n-k+1 \leq i \leq n$. Consider the GRS code $G R S_{k}(\mathbf{a}, \mathbf{v})$ of length $n$ over $\mathbb{F}_{q}$ associated with $\mathbf{a}$ and $\mathbf{v}$, i.e.,

$$
G R S_{k}(\mathbf{a}, \mathbf{v})=\left\{\left(f\left(\alpha_{1}\right), \cdots, f\left(\alpha_{n-k}\right), \gamma f\left(\alpha_{n-k+1}\right), \cdots, \gamma f\left(\alpha_{n}\right)\right) \mid f(X) \in \mathbb{F}_{q}[X], \operatorname{deg} f(X) \leq k-1\right\} .
$$

As in the proofs of the previous theorems, we can show that $G R S_{k}(\mathbf{a}, \mathbf{v}) \bigcap G R S_{k}(\mathbf{a}, \mathbf{v})^{\perp}=\{\mathbf{0}\}$. Indeed, let $\mathbf{c}=\left(f\left(\alpha_{1}\right), \cdots, f\left(\alpha_{n-k}\right), \gamma f\left(\alpha_{n-k+1}\right), \cdots, \gamma f\left(\alpha_{n}\right)\right)$ be an element of $G R S_{k}(\mathbf{a}, \mathbf{v}) \bigcap G R S_{k}(\mathbf{a}, \mathbf{v})^{\perp}$. 
Using Lemma 3.1, we have that a polynomial $g(X) \in \mathbb{F}_{q}[X]$ with $\operatorname{deg} g(X) \leq n-k-1$ can be found such that

$$
\left(f\left(\alpha_{1}\right), \cdots, f\left(\alpha_{n-k}\right), \gamma^{2} f\left(\alpha_{n-k+1}\right), \cdots, \gamma^{2} f\left(\alpha_{n}\right)\right)=\left(u_{1} g\left(\alpha_{1}\right), u_{2} g\left(\alpha_{2}\right), \cdots, u_{n} g\left(\alpha_{n}\right)\right),
$$

where $u_{i}=\prod_{1 \leq j \leq n, j \neq i}\left(\alpha_{i}-\alpha_{j}\right)^{-1}$ for $1 \leq i \leq n$. However, for any $1 \leq i \leq n$ we have

$$
u_{i}=\prod_{1 \leq j \leq n, j \neq i}\left(\alpha_{i}-\alpha_{j}\right)^{-1}=h^{-1} .
$$

Therefore,

$$
\left(f\left(\alpha_{1}\right), \cdots, f\left(\alpha_{n-k}\right), \gamma^{2} f\left(\alpha_{n-k+1}\right), \cdots, \gamma^{2} f\left(\alpha_{n}\right)\right)=\left(h^{-1} g\left(\alpha_{1}\right), h^{-1} g\left(\alpha_{2}\right), \cdots, h^{-1} g\left(\alpha_{n}\right)\right) .
$$

The desired result can be obtained by applying arguments similar to those used in the proof of Theorem 3.4. We are done.

Theorems 3.4 and 3.5 tell us that $k$-dimensional LCD MDS codes of length $q-1$ and $q$ over $\mathbb{F}_{q}$ exist for any $k$, respectively. The following result implies that if the code length $n$ is close to the alphabet size $q$, then LCD GRS codes of length $n$ over $\mathbb{F}_{q}$ exist.

Theorem 3.6. Let $q>3$ be an odd prime power and let $n$ be a positive integer with $1<n<q$. If $1<k \leq\lfloor n / 2\rfloor$ and $n+k \geq q+1$, then there exists a $k$-dimensional LCD GRS code of length $n$ over $\mathbb{F}_{q}$.

Proof. Let $\mathbf{a}=\left(\alpha_{1}, \alpha_{2}, \cdots, \alpha_{n}\right)$, where $\alpha_{i}(1 \leq i \leq n)$ are distinct elements of $\mathbb{F}_{q}$. Label the elements of $\mathbb{F}_{q}=\left\{\alpha_{1}, \alpha_{2}, \cdots, \alpha_{n}, \alpha_{n+1}, \cdots, \alpha_{q}\right\}$. Recall that the dual of $G R S_{k}(\mathbf{a}, \mathbf{1})$ is $G R S_{n-k}(\mathbf{a}, \mathbf{u})$, where $\mathbf{u}=\left(u_{1}, u_{2}, \cdots, u_{n}\right)$ with $u_{i}=\prod_{1 \leq j \leq n, j \neq i}\left(\alpha_{i}-\alpha_{j}\right)^{-1}$. Let

$$
\mathbf{v}=\left(v_{1}, v_{2}, \cdots, v_{q-k}, v_{q-k+1}, \cdots, v_{n}\right),
$$

where $v_{i}=1$ for $1 \leq i \leq q-k$ and $v_{i}, q-k+1 \leq i \leq n$, is chosen such that $-v_{i}^{2} \prod_{j=n+1}^{q}\left(\alpha_{i}-\alpha_{j}\right) \neq u_{i}$. Consider the GRS code $G R S_{k}(\mathbf{a}, \mathbf{v})$ of length $n$ over $\mathbb{F}_{q}$ associated with a and $\mathbf{v}$, i.e.,

$G R S_{k}(\mathbf{a}, \mathbf{v})=\left\{\left(f\left(\alpha_{1}\right), \cdots, f\left(\alpha_{q-k}\right), v_{q-k+1} f\left(\alpha_{q-k+1}\right), \cdots, v_{n} f\left(\alpha_{n}\right)\right) \mid f(X) \in \mathbb{F}_{q}[X], \operatorname{deg} f(X) \leq k-1\right\}$.

We are left to show that $G R S_{k}(\mathbf{a}, \mathbf{v}) \cap G R S_{k}(\mathbf{a}, \mathbf{v})^{\perp}=\{\mathbf{0}\}$. To this end, let

$$
\mathbf{c}=\left(f\left(\alpha_{1}\right), \cdots, f\left(\alpha_{q-k}\right), v_{q-k+1} f\left(\alpha_{q-k+1}\right), \cdots, v_{n} f\left(\alpha_{n}\right)\right)
$$

be an arbitrary element of $G R S_{k}(\mathbf{a}, \mathbf{v}) \cap G R S_{k}(\mathbf{a}, \mathbf{v})^{\perp}$. It follows from Lemma 3.1 that a polynomial $g(X) \in \mathbb{F}_{q}[X]$ with $\operatorname{deg} g(X) \leq n-k-1$ can be found such that

$$
\left(f\left(\alpha_{1}\right), \cdots, f\left(\alpha_{q-k}\right), v_{q-k+1}^{2} f\left(\alpha_{q-k+1}\right), \cdots, v_{n}^{2} f\left(\alpha_{n}\right)\right)=\left(u_{1} g\left(\alpha_{1}\right), u_{2} g\left(\alpha_{2}\right), \cdots, u_{n} g\left(\alpha_{n}\right)\right) .
$$

As a consequence, the first $q-k$ coordinates of (3.5) give $f\left(\alpha_{i}\right)=u_{i} g\left(\alpha_{i}\right)$ for $1 \leq i \leq q-k$; equivalently,

$$
f\left(\alpha_{i}\right)=\prod_{1 \leq j \leq n, j \neq i}\left(\alpha_{i}-\alpha_{j}\right)^{-1} g\left(\alpha_{i}\right)=-\prod_{j=n+1}^{q}\left(\alpha_{i}-\alpha_{j}\right) g\left(\alpha_{i}\right)
$$

where the last equality holds because

$$
\prod_{1 \leq j \leq q, j \neq i}\left(\alpha_{i}-\alpha_{j}\right)=-1 .
$$

This suggests that the polynomial $f(X)+\prod_{j=n+1}^{q}\left(X-\alpha_{j}\right) g(X)$ has $q-k$ distinct roots $\alpha_{1}, \cdots, \alpha_{q-k}$. Note that

$$
\operatorname{deg} f(X) \leq k-1 \leq n-k-1 \leq q-k-1
$$


and

$$
\operatorname{deg} \prod_{j=n+1}^{q}\left(X-\alpha_{j}\right) g(X) \leq(q-n)+(n-k-1)=q-k-1 .
$$

These imply the degree of $f(X)+\prod_{j=n+1}^{q}\left(X-\alpha_{j}\right) g(X)$ is at most $q-k-1$. We thus conclude that

$$
f(X)=-\prod_{j=n+1}^{q}\left(X-\alpha_{j}\right) g(X)
$$

In particular, $\operatorname{deg} f(X)=q-n+\operatorname{deg} g(X) \leq k-1$, giving $\operatorname{deg} g(X) \leq n+k-q-1$. However, the last $n+k-q$ coordinates of (3.5) imply that for any $q-k+1 \leq i \leq n$,

$$
v_{i}^{2} f\left(\alpha_{i}\right)=u_{i} g\left(\alpha_{i}\right)=-v_{i}^{2} \prod_{j=n+1}^{q}\left(\alpha_{i}-\alpha_{j}\right) g\left(\alpha_{i}\right) .
$$

We have $g\left(\alpha_{i}\right)=0$ since $v_{i}$ is chosen such that $-v_{i}^{2} \prod_{j=n+1}^{q}\left(\alpha_{i}-\alpha_{j}\right) \neq u_{i}$. Therefore $g(X)$ has $n+k-q$ distinct roots, which forces $g(X)=0$. We finally conclude that $f(X)=0$, as desired.

We conclude this section with the following theorem.

Theorem 3.7. Let $q>3$ be an odd prime power and let $n$ be a positive integer with $1<n<q$. If $1<k \leq\lfloor n / 2\rfloor$ and $2 n-k<q \leq 2 n$, then there exists a $k$-dimensional LCD GRS code of length $n$ over $\mathbb{F}_{q}$.

Proof. Let $\mathbf{a}=\left(\alpha_{1}, \alpha_{2}, \cdots, \alpha_{n}\right)$, where $\alpha_{i}(1 \leq i \leq n)$ are distinct elements of $\mathbb{F}_{q}$. Label the elements of $\mathbb{F}_{q}=\left\{\alpha_{1}, \alpha_{2}, \cdots, \alpha_{n}, \alpha_{n+1}, \cdots, \alpha_{q}\right\}$. The assumption $2 n-k<q$ is equivalent to saying that $q-n>n-k$. Let $\mathbf{v}=\left(v_{1}, v_{2}, \cdots, v_{n}\right)$ with

$$
v_{i}=\prod_{j=1}^{n-k}\left(\alpha_{i}-\alpha_{n+j}\right)
$$

for $1 \leq i \leq n$. Consider the GRS code $G R S_{k}(\mathbf{a}, \mathbf{v})$ of length $n$ over $\mathbb{F}_{q}$ associated with $\mathbf{a}$ and $\mathbf{v}$, i.e.,

$$
G R S_{k}(\mathbf{a}, \mathbf{v})=\left\{\left(v_{1} f\left(\alpha_{1}\right), v_{2} f\left(\alpha_{2}\right), \cdots, v_{n} f\left(\alpha_{n}\right)\right) \mid f(X) \in \mathbb{F}_{q}[X], \operatorname{deg} f(X) \leq k-1\right\} .
$$

We claim that $G R S_{k}(\mathbf{a}, \mathbf{v}) \bigcap G R S_{k}(\mathbf{a}, \mathbf{v})^{\perp}=\{\mathbf{0}\}$. To this end, let

$$
\mathbf{c}=\left(v_{1} f\left(\alpha_{1}\right), v_{2} f\left(\alpha_{2}\right), \cdots, v_{n} f\left(\alpha_{n}\right)\right)
$$

be an arbitrary element of $G R S_{k}(\mathbf{a}, \mathbf{v}) \cap G R S_{k}(\mathbf{a}, \mathbf{v})^{\perp}$. It follows from Lemma 3.1 that a polynomial $g(X) \in \mathbb{F}_{q}[X]$ with $\operatorname{deg} g(X) \leq n-k-1$ can be found such that

$$
\left(v_{1}^{2} f\left(\alpha_{1}\right), v_{2}^{2} f\left(\alpha_{2}\right), \cdots, v_{n}^{2} f\left(\alpha_{n}\right)\right)=\left(u_{1} g\left(\alpha_{1}\right), u_{2} g\left(\alpha_{2}\right), \cdots, u_{n} g\left(\alpha_{n}\right)\right) .
$$

This gives $v_{i}^{2} f\left(\alpha_{i}\right)=u_{i} g\left(\alpha_{i}\right)$ for $1 \leq i \leq n$. By the definition of $v_{i}$,

$$
v_{i}^{2} f\left(\alpha_{i}\right)=\prod_{j=1}^{n-k}\left(\alpha_{i}-\alpha_{n+j}\right)^{2} f\left(\alpha_{i}\right) .
$$

On the other hand,

$$
u_{i} g\left(\alpha_{i}\right)=\prod_{1 \leq j \leq n, j \neq i}\left(\alpha_{i}-\alpha_{j}\right)^{-1} g\left(\alpha_{i}\right)=-\prod_{j=n+1}^{q}\left(\alpha_{i}-\alpha_{j}\right) g\left(\alpha_{i}\right) .
$$

These lead to

$$
\prod_{j=1}^{n-k}\left(\alpha_{i}-\alpha_{n+j}\right) f\left(\alpha_{i}\right)=-\prod_{j=n+(n-k+1)}^{q}\left(\alpha_{i}-\alpha_{j}\right) g\left(\alpha_{i}\right)
$$


for $1 \leq i \leq n$. At the moment we have

$$
\operatorname{deg}\left(\left(\prod_{j=1}^{n-k}\left(X-\alpha_{n+j}\right)\right) f(X)\right) \leq(n-k)+(k-1)=n-1
$$

and

$$
\operatorname{deg}\left(\left(\prod_{j=n+(n-k+1)}^{q}\left(X-\alpha_{j}\right)\right) g(X)\right) \leq(q-n)-(n-k)+(n-k-1)=q-n-1 \leq n-1,
$$

where the last inequality holds because $q \leq 2 n$ by our assumption. Hence the degree of

$$
\left(\prod_{j=1}^{n-k}\left(X-\alpha_{n+j}\right)\right) f(X)+\left(\prod_{j=n+(n-k+1)}^{q}\left(X-\alpha_{j}\right)\right) g(X)
$$

is at most $n-1$. We thus conclude from (3.6) that

$$
\left(\prod_{j=1}^{n-k}\left(X-\alpha_{n+j}\right)\right) f(X)=-\left(\prod_{j=n+(n-k+1)}^{q}\left(X-\alpha_{j}\right)\right) g(X) .
$$

Obviously $\prod_{j=1}^{n-k}\left(X-\alpha_{n+j}\right)$ is a divisor of $g(X)$. This forces $g(X)=0$, as $\operatorname{deg} g(X) \leq n-k-1$. We are done.

Acknowledgements The research of Bocong Chen was supported by NSFC (Grant No. 11601158). The research of Hongwei Liu was supported by NSFC (Grant No. 11171370) and self-determined research funds of CCNU from the collegess basic research and operation of MOE (GrantNo. CCNU14F01004).

\section{References}

[1] S. Ball, Finite Geometry and Combinatorial Applications, London Mathematical Society Student Texts 82, Cambridge University Press, 2015.

[2] C. Carlet and S. Guilley, Complementary dual codes for counter-measures to side-channel attacks, Adv. Math. Commun., vol. 10, pp. 131-150, 2016.

[3] H. Q. Dinh, Structure of repeated-root cyclic and negacyclic codes of length $6 p^{s}$ and their duals, Contemp. Math., vol. 609, pp. 69-87, 2014.

[4] S. T. Dougherty, J.-L. Kim, B. Ozkaya, L. Sok and P. Solé, The combinatorics of LCD codes: Linear programming bound and orthogonal matrices, arXiv:1506.01955, 2015.

[5] M. Esmaeili and S. Yari, On complementary-dual quasi-cyclic codes, Finite Fields Appl., vol. 15, pp. 375-386, 2009.

[6] C. Güneri, B. Özkaya and P. Solé, Quasi-cyclic complementary dual codes, Finite Fields Appl., vol. 42, pp. 67-80, 2016.

[7] X. Hou and F. Oggier, On LCD codes and lattices, in Proc. IEEE Int. Symp. Inf. Theory, pp. $1501-1505,2016$.

[8] L. Jin, Construction of MDS codes with complementary duals, IEEE Trans. Inf. Theory, DOI 10.1109/TIT.2016.2644660, 2016.

[9] L. Jin and C. Xing, New MDS self-dual codes from generalized Reed-Solomon codes, IEEE Trans. Inf. Theory, DOI 10.1109/TIT.2016.2645759, 2016. 
[10] C. Li, C. Ding and S. Li, LCD cyclic codes over finite fields, arXiv:1608.02170, 2016.

[11] C. Li, C. Ding and H. Liu, Parameters of two classes of LCD BCH codes, arXiv:1608.02670, 2016.

[12] S. Li, C. Ding and H. Liu, A family of reversible BCH codes, arXiv:1608.02169, 2016.

[13] S. Ling and C. Xing, Coding Theory: A First Course, Cambridge University Press, Cambridge, 2004.

[14] F. J. MacWilliams and N. J. A. Sloane, The Theory of Error-Correcting Codes, Elsevier, NorthHolland, 1977.

[15] J. L. Massey, Linear codes with complementary duals, Discrete Math., vol. 106-107, pp. 337-342, 1992.

[16] S. Mesnager, C. Tang and Y. Qi, Complementary dual algebraic geometry codes, arXiv:1609.05649, 2016.

[17] M. Sari and M. E. Köroğlu, On MDS negacyclic LCD codes, arXiv:1611.06371, 2016.

[18] X. Yang and J. L. Massey, The necessary and sufficient condition for a cyclic code to have a complementary dual, Discrete Math., vol. 126, pp. 391-393, 1994.

[19] S. Zhu, B. Pang and Z. Sun, The reversible negacyclic codes over finite fields, arXiv:1610.08206, 2016. 